\title{
Doctor Recommendation Chatbot: A research study
}

\author{
Umar Jameel1, ${ }^{\text {, }}$, Hashim Khan², Aqib Anwar ${ }^{2}$ \\ ${ }^{1} \mathrm{CS}$ and IT, The University of Lahore (Islamabad campus), Rawalpindi 44000, Pakistan \\ ${ }^{2} \mathrm{CS}$ and IT, The University of Lahore (Islamabad campus), Islamabad 46000, Pakistan
}

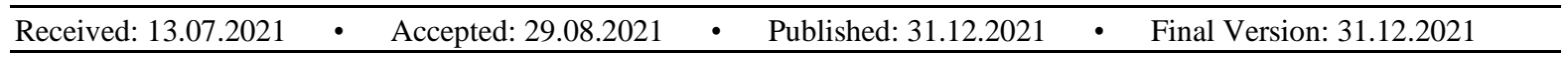

\begin{abstract}
Healthcare is very important in our life. As the population increases, day by day the quality of communication between patients and doctors becomes less effective. So because of a load of patients in hospitals, the quality of treatment decreases day by day. So, there should be a system by which a patient can easily communicate with a chatbot. Then the chatbot will examine the patient's problem and at last, the chatbot will recommend a specific doctor for a patient. With the help of artificial intelligence, the proposed system will be developed using natural language processing that means the chatbot first diagnoses the patient's problem then recommends a doctor for consultation. During the COVID pandemic, the trends of virtual assistance increased, and people are interacting with robots and virtual assistants. In many countries, there are robots in hospital receptionists to solve problems of people and work like humans. Chatbots make it easy to find a particular doctor by telling them problems. In this research study, there are different literature reviews included making a healthcare chatbot.
\end{abstract}

Keywords: Healthcare, Chatbot, Healthcare Chatbot, NLP, Artificial Intelligence

\section{Introduction}

We can see a lot of changes in hospitals and healthcare systems in the past few years. The change in Artificial Intelligence increased and people are moving to interact with virtual assistance and robots for communication because of their best quality. Doctor Recommendation chatbot, a system which can be used by patients to get recommendations for a doctor. In this system the patient can tell the problem to the chatbot and the chatbot will use its algorithm to solve the patient problem by recommending a particular doctor to him. Like a patient telling his problem to a chatbot, chatbot then starts processing algorithms to find the perfect solution for the patient then recommends a doctor as a result. The chatbot will use the answer question box to communicate with patients. Patient tells his/her problem to the chatbot using text, then the chatbot will respond to the problem using a query.

\subsection{History of chatbot}

Chatbots of different types like business, healthcare were introduced in recent years because of demand and popularity and the increase of technology and Artificial Intelligence. But chatbot was not made in the past year as it was first made in the early 1960's. The name of this chatbot was "Eliza". But it didn't catch and gain so much popularity because people of that time were unaware of such technology. Later on in 1972 "parry" chatbot was introduced, it was a healthcare chatbot developed by an American psychiatrist K.Colby. In the 1990s, Jabberwacky and dr.sbaitso were introduced. Later on in 1995 Richard Walace developed a chatbot named ALICE that was using

\footnotetext{
* Corresponding Author: $70065581 @$ student.uol.edu.pk
} 
universal language processing. But later on in 2009 a Chinese company introduced "WeChat", it gained more popularity among people. So, the trend of chatbots started from there and later on researchers and different technology organizations started working on it. After that Apple developed a chatbot for IOS users which was named "Siri" in 2010. It was deeply integrated with different types of things like audio, video and text etc. In 2012 Google now was developed by Google and in 2014 Microsoft launched their chatbot cortana which was using voice recognition. In 2014 after Cortana , Alexa was developed by amazon and now many platforms integrate it into their systems. In 2016 different social media platforms introduced artificial technology data and different types of robots by natural language processing and artificial intelligence.

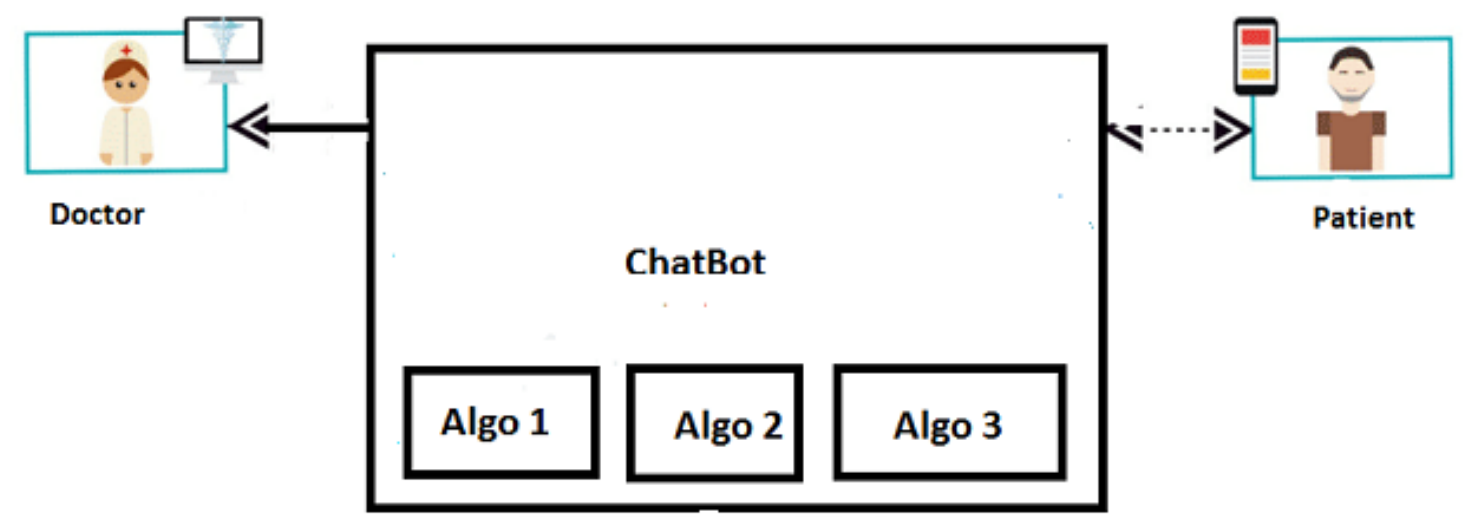

Figure 1. Chatbot

The working of the chatbot algorithm is based on natural language processing which means a user will enter his query and then chatbot will further process the patient's query to understand and then try to fetch some relevant keywords for best response. A user can get a recommendation when he tells his symptoms to the chatbot and chatbot will diagnose the user problem according to symptoms and then recommend a doctor for consultation using zip code. Zip code is different for each city so there can be many doctors available. So, after entering a zip code the user will see only the nearest available doctor for consultation.

The trends of chatbots increase with the increase in technology. Many platforms now include virtual assistance for users or customers to solve their minor problems which can save their time and money also. During the COVID pandemic when shortage of employees occurred then people introduced different types of chatbots like virtual assistant chatbot, AI chatbot, gaming chatbot and healthcare chatbots. You can see the graph of the trend of chatbots. 


\section{Chatbot adoption across different industries}

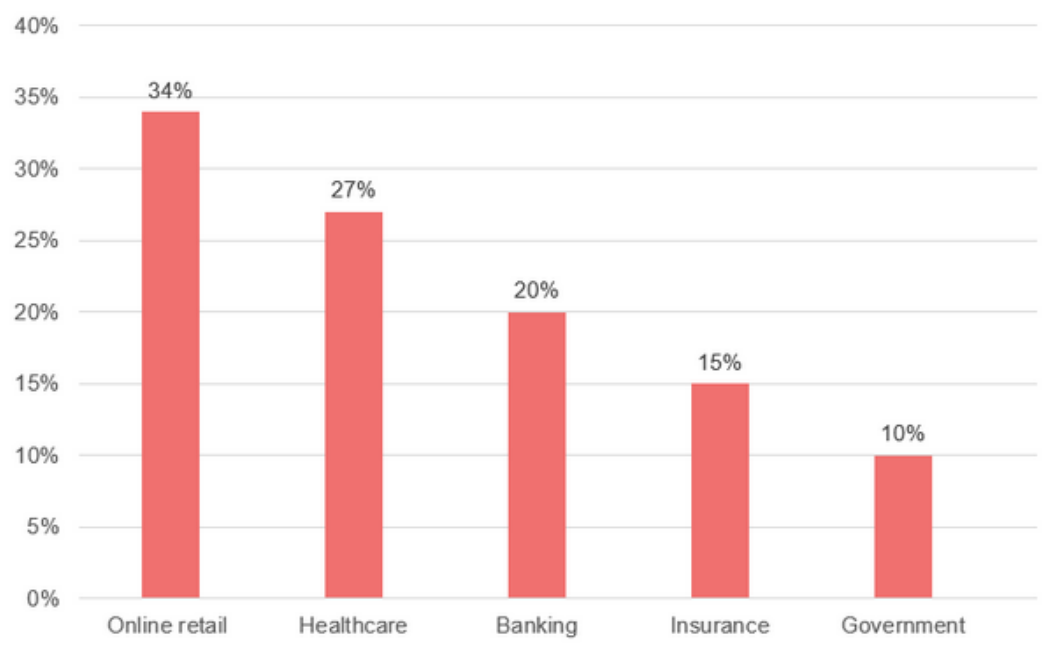

Figure 2. Graph of chatbot adoption

You can see that people are now moving to interact with chatbots in different industries. And the future of chatbots is also bright as you can see in this graph.

\section{The future of chatbots}

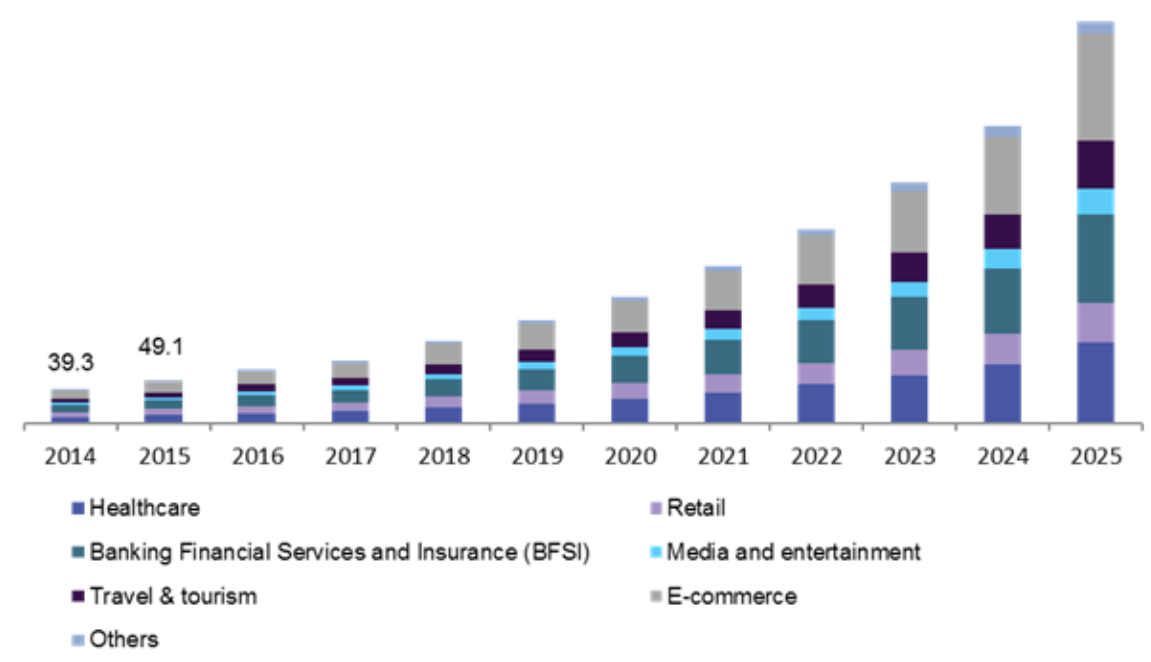

Figure 3. The future of chatbots 


\section{Literature Review}

\subsection{Healthcare Chatbot}

Many scholars have already given research on healthcare chatbots and their trends in the market. In this paper there are few of them added for our research study. The chatbot is defined as one of the most advanced and promising ways of communicating between humans and machines. From a technological point of view it is a question answer system but here computers are involved on one side, in short the chatbots are machine-controlled systems that replicate users behavior on one facet of the chatting communication. They are mimic systems that imitate the conversations between more than one individual. they supply a simulating platform for effective and sensible communications with the user on the opposite finish. [1]

They copy marketers, sales persons, counsellors and alternative mediators and work to supply services that the higher than mentioned folks provide. There are wide ranges of chatbots catering in several domains a number of them are as follows: business, market, stock, client care, healthcare, counselling, recommendation systems, web, entertainment, brokering, journalism, on-line food and accessory searching, travel chatbots, banking chatbots, recipe guides, etc. the foremost famed chatbots like Alexa or Google assistant ar the most effective examples which will tend for sensible communicating chatbots. These are general purpose chatbots that give services for all domains and don't seem to be restricted to a specific domain. There are domain specific chatbots which provide functionalities to the higher than mentioned domains. [2]

Some of them are as follows: Botsify may be a chatbot that helps developers to form sensible Facebook traveler Chatbots and is employed to gather info from Facebook users. Imperson may be a chatbot that helps developers to form business chatbots and supply client care services. NBC is a chatbot that helps the newsreaders to navigate fastly through high headlines. [3]

We are writing this paper to make some proposals, to give a plan to overcome the individuals' problems during the isolation period so that one cannot come in direct contact or interface the clinical specialists distantly and self-personalize from Covid 19. The pandemic spread right around 200 territories, made the entire population of the world vulnerable, and killed more than 5 million individuals and left billions in isolation. So here our chatbot can make a big difference so that individuals are not too stressed over to go clinics or dispensaries. [4]

\subsection{Role of AI and Natural language processing in chatbots}

Artificial Intelligence and natural language processing plays a very important role in designing a chatbot. How will our chatbot system work? For starters our system would consist of natural language generation and retrieval models along with that it would also include template-based models, sequence-to-sequence and latent variable neural network models. By applying reinforcement for learning for crowdsourcing data and real-world user interactions, this system of chatbot has been trained to select a valid and responsible response from the models in its ensemble with a 99 percent validation rate. [5]

The design of the chatbot system that we have proposed will interact with users with the help of a web based platform in which a user will register Once set up is done chatbot will answer based on the diseases and last medications, ask for symptoms and answer causes and prevention or medical suggestions in the form of medicine. [6]

No doubt it is the age of computing and computers play an important role in our society, it not only gives us information but also is the source of entertainment to us and helps us in various other 
matters. We are well familiar with chatbots. It is a program designed to mimic a smart person that communicates on a text or spoken ground.[7] Chatbot recognizes the user input by either pattern matching or by predefined acknowledgments like frequently asked questions are fed into the dataset for replies or interaction with users. For instance, if the user is providing the bot a sentence like "Who are you?" The chatbot can reply to you as "I am Chatbot." Or like the chatbot replies as "I can be called Chatbot., Hi I am your assistant, I am a chatbot etc". The questions and answers are solely based on the interaction given by the user. The response generated by the chatbot totally depends upon the $\mathrm{Q}$ and $\mathrm{A}$ of the user and after evaluation by the chatbot a response is generated from a predefined pattern to satisfy a user. Pattern comparison is done in a chatbot for implementation, after which the sequence or order of text or sentence is defined or recognized so that a saved response pattern is acclimatized to the variables exclusive to the sentence.[8] Our chatbot system is not highly sophisticated engineering marvel it cannot register and respond to questions with high complexity, and are unable to perform compound activities. Chatbots as we know are relatively new technology. It has a long way to go and the application of a Chatbot can be seen in various fields in the future. In our project we will cover the design and implementation of a chatbot. The key purpose of chatbot is not to come directly in contact with the doctor(face to face) but in today's world when we all are in the middle of pandemic due to COVID-19 it has become the new norm of today's world to avoid physical contact at all costs to prevent pandemic.[9]

While it is necessary to promote health and safety of patients, the hos pital also has to protect its employees so chatbots come in as a very useful tool for interaction between hospitals and patients. Chatbots are programmed in such a way which can interact with patient by text, voice and animation.[10] As the health department or hospitals are not equipped to face pandemics on such a greater scale it doesn't have the resources to perform thousands of symptoms around the clock and screens daily. So, in order to tackle the issue to prevent pandemic and follow and fulfill all the SOPs and guidelines issued by World Health Organization, chatbot is the first line of defense for both patients as well as health workers and most of all to prevent overcrowding of Hospitals. Chatbot is a dynamic tool that enables screening and prevents the spread of Covid-19 pandemic. And there is an increasing barrier between patients and doctor communication because of the load of patients, they can't get timely advice on their medicine. [11]

Chatbots have changed the communication methodology because when there were no computers in the world people communicated with each other in different perspectives, but now technology grows and users are interacting with digital machines and bots for conversation. It is done by natural language processing which makes us able to communicate with robots.[12] In all of the world clinical and health systems or hospitals face problems and pressures because of change in the world, demographics change and change in technology. Which demands to integrate different things with clinics and hospitals like, bots, AI, and smart hospitals etc. Because nowadays health systems are moving analog to digital for efficient and best results of treatments.[13] The chatbot system interacts with the patient in the healthcare system in such a way which can identify disease by calculating different type of symptoms and based on the query they respond to the user.[14] It is important for cancer patients that their treatment is led by professionals and in a control secured environment to normalize the stress reactions and discuss the fear of participants through online chat to keep their mental health and stress low as in majority cases the cancer patient loses its will of living upon coming to know of cancer. [15]

Chatbots have been a lifesaver ever since its introduction or induction into the field of medical, not only it provides patients with resources regarding mental health but also with lifesaving or critical information to the patients. [16] The demand of chatbots skyrocketed in the current Covid-19 
pandemic due to social distancing which flooded the market with a variety of chatbots that includes or focuses on both simple and complex activities like behavior changing, life support, monitoring of health etc. to name a few. [17]

Chatbots when combined with Artificial Intelligence can save both time and money. Also it is very effective and with growing demand it has seen a lot of surge in the past year or so due to the global pandemic situation. [18] The whole idea behind the introduction of chatbot to the field of medical is to avoid unnecessary contact by carrying virtual doctors in our pockets and most of all the freedom of consulting a doctor at any given time or place. It has the ability to bring a doctor at your doorsteps whenever you want "Literally". [19]

\section{Critical Review on Literature}

In my opinion all researchers provided the best data on their own. Everyone proposed their own system regarding chatbots for healthcare. They did research and then gave an idea to model a system. I think chatbot systems play a great role as virtual assistance in the business field and in healthcare fields. Because the chatbot system reduces the workload of doctors by communicating with patients.I think Artificial Intelligence and Natural language Processing (NLP) plays a great role in making a healthcare chatbot because the system first needs to understand the human language and then try to respond. For this purpose NLP will be used. To model chatbot there will be algorithms used in backend like Term Frequency and inverse data frequency for best word selection from query.

\section{System Architecture}

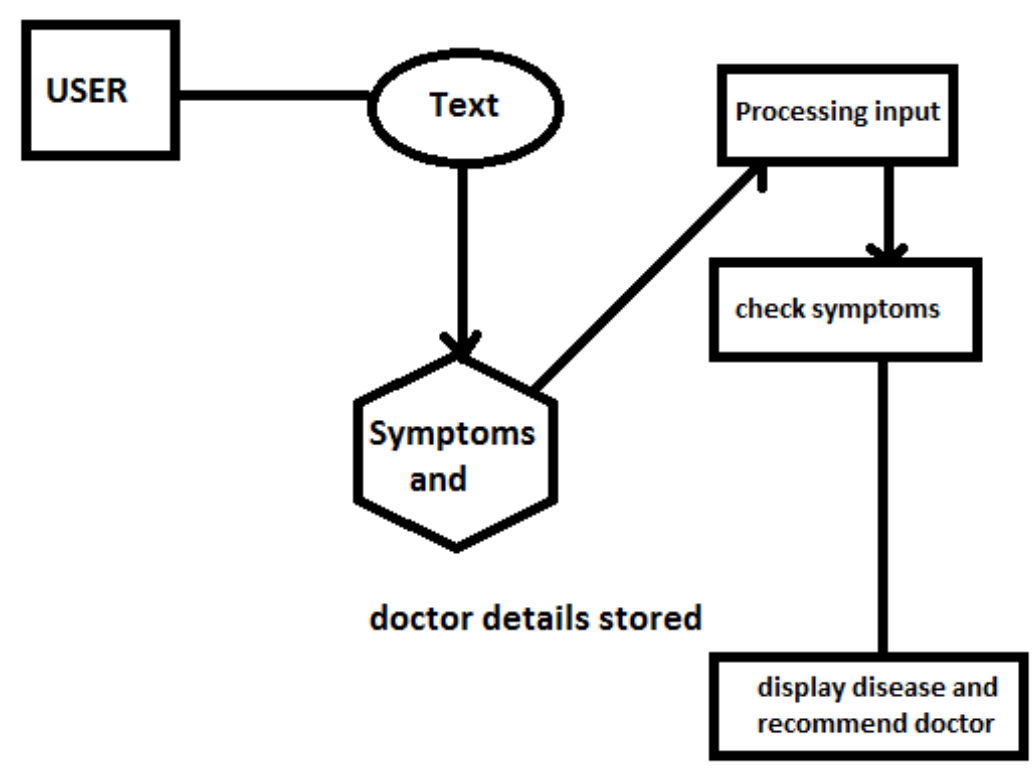

Figure 4. System Architecture

By using this architecture developers can develop chatbots. There will be a system in which a user can interact with bot for chatting and enter his text. Then the chatbot will calculate symptoms of the disease which the patient introduced. Now it is the stage of processing data which uses algorithms and natural language processing. Then the system will display the answer of the patient query. 


\section{Methodology}

This chatbot system is a healthcare system and it will contain different types of symptoms to calculate disease. So natural language processing will be done to communicate with the bot and then the bot will answer our query. TF-IDF algorithm will be used to make this chatbot, TF means term frequency and IDF means inverse document frequency. This algorithm is used to calculate the high prediction or frequency by calculating the number of words in sentences and number of words in a document. This algorithm will be helpful to design healthcare chatbot because it will calculate the user queries by matching words from symptoms and will find the best solution for it. Cosine similarity can also be used with term frequency.

\section{Conclusion}

The healthcare chatbot is a great tool for communication between patient and bot. With the help of chatbot doctors can save their time for minor problems and it also helps patients to solve their issues. A patient can have a conversation with a bot using text and the chatbot will respond instantly without any delay. The trends of chatbots are increasing day by day with the passage of time because of the load of patients in hospitals.

\section{References}

[1] S.T.A.R.S. Nivedita Bhirud, "Chatbots In Healthcare," INTERNATIONAL JOURNAL OF SCIENTIFIC \& TECHNOLOGY, vol. 8, no. 7, p. 7, July 2019.

[2] W.-K. Chen, Linear Networks and Systems (Book style).Belmont, CA: Wadsworth, 1993, pp. 123-135.

[3] F. A. K. S. J. N. Cyril Joe Baby, "ChatBot using natural Language Processing," Automation using IOT, 4 January 2018.

[4] D. L. B. K. H.-J. C. Kyo-Joong Oh, "A Chatbot for Psychiatric Counseling in Mental Healthcare Service Based on Emotional Dialogue Analysis and Sentence Generation," 2017 18th IEEE International Conference on Mobile Data Management (MDM), July 2017.

[5] C. K. S. V. M. S. D. Balasubramaniam, "DESIGN AND DEVELOPMENT OF SMART HEALTHCARE CHATBOT APPLICATION USING AI-ML," Journal of Natural Remedies, vol. 21, pp. 13-20, 2020.

[6] C. S. M. G. S. Z. Z. L. Iulian V. Serban, "A Deep Reinforcement Learning Chatbot," in Montreal Institute for Learning Algorithms, Montreal, Quebec, Canada, 5 November 2017.

[7] S. S. S. N. V. K. P. V. S. G. Ojas Kadu, "Intelligent Healthbot for Transforming Healthcare," International Journal of Trend in Scientific Research and Development (IJTSRD), vol. 3, no. 3, pp. 1576-1579, MarchApril 2019.

[8] M. Dahiya, "A Tool of Conversation: Chatbot," International Journal of Computer Sciences and Engineering, vol. 5, no. 5, pp. 159-161, 30 May 2017.

[9] A.Y.O.Y.O.D.S.R.G. a. A. B. N. Timothy J. Judson, "Implementation of a digital Chatbot to screen health system employees during the covid-19 pandemic," Journal of the American Medical Informatics Association, vol. 27, pp. 1450-1455, 12 June 2020.

[10] A.A.M.N.K.D.B Mowafa, "Perceptions and opinions of patients about mental health chatbots", Vol 23, issue 1, el 7828. 2021

[11] X.Fan, Z. Zhang, D. Chao, F.Tian, "Utilization of Self-Diagnosis Health Chatbots in Real-World Settings:Case Study", Vol 23, issue 1, el 9928, 2021

[12] B.A Shawa, E. Atwell, "Different measurements metrics to evaluate a chatbot system", April 2007

[13] K. Saligrama, P.Shetty, "AI Enabled Healthcare Chatbot Systems for Hospital Web Applications”, Volume 3 , issue 5, May 2020 
[14] K.B.R, Dr. Cheetana, "Chatbot for healthcare system using Artificial Intelligence”, Volume 5, issue 3, ISSN 2454-132X, p 1304

[15] E. W. A. A. G. H. D. d. S. J. W. J. L. B. M. G. D. G. D. A. M. J. E. Yvonne W Leung, "Natural Language Processing-Based Virtual Co Facilitator for Online cancer support groups: Protocol for an Algorithm Development and Validation Study," JMIR RESEARCH PROTOCOLS, vol. 10, no. 1.

[16] M. Zeineb Safi, P. Alaa Abd-Alrazaq, P. Mohamed Khalifa and P. Mowafa Househ, "Technical Aspects of Developing Chatbots for Medical Applications: Scoping Review," JOURNAL OF MEDICAL INTERNET RESEARCH, vol. 22, no. 12, December 2020.

[17] C. d. C. E. L. M. H. S. N. d. P. G. M. E. N. E. M. Madison Milne-Ives, "The Effectiveness of Artificial Intelligence Conversational Agents in Health Care: Systematic Review," JOURNAL OF MEDICAL INTERNET RESEARCH, vol. 22, no. 10, December 2020.

[18] Y. J. O. P. L. Z. Y. Y. F. Jingwen Zhang, "Artificial Intelligence Chatbot Behavior Change Model for Designing Artificial Intelligence Chatbots to Promote Physical Activity and a Healthy Diet: Viewpoint," JOURNAL OF MEDICAL INTERNET RESEARCH, vol. 22, no. 9, September 2020.

[19] A. B. A. M. K. M. Krishnendu Rarhi, "Automated Medical Chatbot", SSRN 3090881, 2017 